\title{
Active tuned mass damper for damping of offshore wind turbine vibrations
}

\author{
Brodersen, Mark Laier; Bjørke, Ann-Sofie; Høgsberg, Jan Becker
}

Published in:

Wind Energy

Link to article, DOI:

10.1002/we.2063

Publication date:

2017

Document Version

Peer reviewed version

Link back to DTU Orbit

Citation (APA):

Brodersen, M. L., Bjørke, A-S., \& Høgsberg, J. B. (2017). Active tuned mass damper for damping of offshore wind turbine vibrations. Wind Energy, 20(5), 783-796. https://doi.org/10.1002/we.2063

\section{General rights}

Copyright and moral rights for the publications made accessible in the public portal are retained by the authors and/or other copyright owners and it is a condition of accessing publications that users recognise and abide by the legal requirements associated with these rights.

- Users may download and print one copy of any publication from the public portal for the purpose of private study or research.

- You may not further distribute the material or use it for any profit-making activity or commercial gain

- You may freely distribute the URL identifying the publication in the public portal

If you believe that this document breaches copyright please contact us providing details, and we will remove access to the work immediately and investigate your claim 


\title{
ACTIVE TUNED MASS DAMPER FOR DAMPING OF OFFSHORE WIND TURBINE VIBRATIONS
}

\author{
MARK L. BRODERSEN ${ }^{(1,2)}$, ANN-SOFIE BJØRKE ${ }^{(1,2)}$ AND JAN HØGSBERG $^{(1)}$ \\ (1) DEPARTMENT OF MECHANICAL ENGINEERING, TECHNICAL UNIVERSITY OF DENMARK, \\ DK-2800 KGS. LYNGBY, DENMARK \\ ${ }^{(2)}$ NATIONAL OILWELL VARCO DENMARK I/S, PRIORPARKEN 480, DK-2605 BRØNDBY, DENMARK
}

\begin{abstract}
An Active Tuned Mass Damper (ATMD) is employed for damping of tower vibrations of fixed offshore wind turbines, where the additional actuator force is controlled using feedback from the tower displacement and the relative velocity of the damper mass. An optimum tuning procedure equivalent to the tuning procedure of the passive Tuned Mass Damper (TMD) combined with a simple procedure for minimizing the control force is employed for determination of optimum damper parameters and feedback gain values. By time domain simulations conducted in an aeroelastic code it is demonstrated that the ATMD can be used to further reduce the structural response of the wind turbine compared to the passive TMD, and this without an increase in damper mass. A limiting factor of the design of the ATMD is the displacement of the damper mass, which for the ATMD increases to compensate for the reduction in mass.
\end{abstract}

\section{INTRODUCTION}

The monopile support is currently the most used support structure for offshore wind turbines, primarily due to its simple design. The design is relatively cost-effective compared to for example a jacket structure, though also quite sensitive to wave loading. Dimensioning of the monopiles are usually dictated by the fatigue loads, caused by wind and waves exciting primarily a combination of the two lowest tower modes, the fore-aft mode in the rotor direction and the side-side mode lateral to the rotor direction. Waves acting with an angle to the wind can therefore cause large fatigue damage due to the absence of the aerodynamic damping in the direction lateral to the wind $[1,2,3]$. The future will see an increasing number of larger and more slender wind turbines positioned at deeper water depths [4]. For these structures the natural frequencies of the critical tower modes will be lower than for present day wind turbine structures, which will cause fatigue damage due to resonant wave loading to increase significantly. One way to reduce fatigue damage due to wave loading, and thereby increase the feasibility of the monopile support, would be to install external dampers.

So far research on the use of external dampers for fixed offshore wind turbines has focused mainly on passive concepts, with most focus on Dynamic Vibration Absorber (DVA) concepts, such as a Tuned Liquid Column Damper (TCLD) or a Tuned Mass Damper (TMD). Some of the pioneering work concerning applications in wind turbines includes the article by Colwell and Basu [5] in which the damping effect of a TLCD installed in an offshore wind turbine has been investigated by assuming correlated wind and wave load conditions, whereas the potential of using a pair of TMDs simultaneously targeting the dominant fore-aft and side-side modes has been demonstrated 
by Lackner and Rotea [6]. More recently, attention has focused on how to address the absence of aerodynamic damping in the side-side direction, when significant wind-wave misalignment is present, e.g. in the work by Stewart and Lackner [7] where the beneficial effect of a TMD in reducing the tower base moment is demonstrated through numerical simulations, and where in particular a significant reduction in the side-side moment has been reported.

In order for a DVA to be effective it needs to be installed where the amplitude of the critical tower mode is largest, which for the wind turbine is at the top of the tower or inside the nacelle. Effective damping by a DVA is furthermore associated with large damper mass, which constitutes a major limitation, since additional mass is highly undesirable at the top of the wind turbine. The Active Tuned Mass Damper (ATMD) is a hybrid device consisting of a passive TMD supplemented by an actuator parallel to the spring and damper. It is a well known concept in structural control, especially for mitigation of excessive dynamic response of high-rise buildings subjected to strong wind and earthquake loads, where the ATMD has been proven to yield enhanced damping performance compared to the passive TMD $[8,9]$. The concept has also been implemented in a number of highrise buildings in Japan [10]. For optimal feedback control of the ATMD, different strategies have been proposed. Chang and Soong [11] proposed a state feedback scheme combined with a Linear Quadratic Regulator (LQR) determined by minimizing both the primary structural response and the control effort, Chang and Yang [12] assumed a white noise excitation and used a complete feedback scheme in which the optimum gain parameters are determined from a minimization of the response variance, while Nishimura [13] proposed a pure acceleration feedback control law, in which the optimum gain is determined by a dynamic amplification analysis similar to that of the passive TMD. As demonstrated in [13], for a single degree of freedom (DOF) system, this acceleration feedback scheme provides a reduction of the dynamic amplification of the primary mass compared to the TMD, but for the same damper mass and without any increase in the maximum dynamic amplification of the damper mass. However, for a flexible structure with a sufficiently large number of DOFs this scheme contains an inherent instability as the mass matrix becomes indefinite in the high-frequency limit, which makes it unfeasible for practical implementation.

The present paper applies the ATMD concept for damping of wind turbine tower vibrations in order to overcome the limitations of the passive TMD, for which effective damping is associated with large damper mass. The application and potential of ATMDs in (off-shore) wind turbines has been demonstrated by Fitzgerald et al. for damping of blade vibrations [14] as well as the global vibrations with emphasis on structure-soil interaction [15]. In [14, 15] effective vibration mitigation is reported for active forces derived on the basis of optimal LQR control, which requires some type of observer to estimate the dynamic state of the structure in real time. In the analysis by Lackner and Rotea [16] the proportional gain associated with modern optimal control is instead derived by solving an $H_{\infty}$ problem, which is conditionally stable and provides effective damping performance when properly tuned. The performance and comparison of TMD and ATMD for damping of floating off-shore wind turbines has furthermore been investigated by Namik et al. [17]. A recent review paper by Rahman et al. [18] provides a discussion of the performance of 
(a)

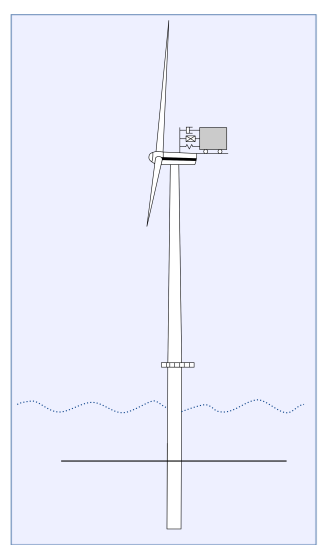

(b)

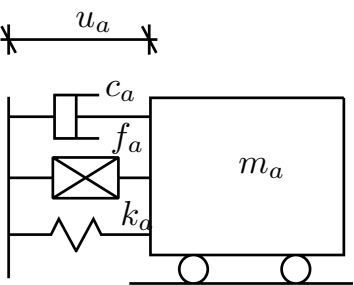

FIgURE 1. (a) Fixed offshore wind turbine with (b) ATMD.

passive and active tuned mass damper systems installed in wind turbines. This shows that the optimal control based on LQR or $H_{\infty}$ optimization is a seemingly preferred approach for the development of active control laws used in mass damper systems.

A simple feedback control equation that provides guaranteed feedback stability also for a flexible structure with multiple DOFs is proposed in Section 2. It is composed of a proportional feedback term to mitigated the structural vibration amplitude and a collocated velocity feedback term to reduce the active control force at the targeted resonance frequency. By assuming a modal representation for the critical tower mode as in Section 2.1 the tuning procedure can be carried out for a reduced 2-DOF system. Applying a dynamic amplification tuning procedure similar to that of the TMD combined with a tuning strategy for reduction of the active control energy, optimal parameters are determined that both give an increased damping performance compared to the passive TMD, but for the same damper mass, and also reduces the required active control forces, as demonstrated in Sections 2.2-2.4. In Section 3 the performance of the proposed ATMD is investigated for damping of wind turbine tower vibrations by the use of comprehensive time domain simulations of a $5 \mathrm{MW}$ offshore reference turbine conducted in the advanced aeroelastic code HAWC2, see [19]. In order to compute the optimum damper parameters the critical tower modes are determined from the free decay response in Section 3.1. Next in Sections 3.2-3.3 it is demonstrated through both a frequency and a time transient response analysis that compared to the passive TMD the present ATMD can further mitigate the response of the wind turbine, without increasing damper mass. Thus, the proposed ATMD comprises guaranteed stability, explicit calibration formulae based on the desired level of dynamic amplification and adjustable control force amplitude. Furthermore, the simple control equation requires the nacelle displacement and the relative damper mass velocity as sensor input, whereby the full state observer associated with optimal LQR control is not needed. 


\section{Modeling of Active Tuned Mass Damper}

A fixed offshore wind turbine structure with an Active Tuned Mass Damper (ATMD) is illustrated in figure 1(a). The ATMD is positioned at the top of the wind turbine, where the amplitude of the critical tower modes are expected to be largest. In the initial design process, the wind turbine structure is assumed to be undamped and described by a linear discrete model with mass matrix $\mathbf{M}$ and stiffness matrix $\mathbf{K}$. This is a fairly valid assumption since the critical tower modes are nearly unaffected by the rotations of the blades and since the modes have very low damping. With reference to figure $1(\mathrm{~b})$ the ATMD exerts a resulting force on the tower

$$
f=c_{a} \dot{u}_{a}+k_{a} u_{a}-f_{a}
$$

where $u_{a}$ is the relative displacement of the damper mass, $f_{a}$ is the force from the active actuator, while $c_{a}$ and $k_{a}$ are the viscous parameter and stiffness of the ATMD, respectively. Hereby the equation of motion (EOM) for the structural DOFs of the wind turbine in $\mathbf{u}$ can be written as

$$
\mathbf{M u ̈}+\mathbf{K u}=\mathbf{f}+\mathbf{w}\left(c_{a} \dot{u}_{a}+k_{a} u_{a}-f_{a}\right) .
$$

In this equation the vector $\mathbf{f}$ represents external loading, while the connectivity vector w defines the connection of the ATMD to the tower. The connectivity vector $\mathbf{w}$ will contain zeros except for the unit value at the DOF, at which the ATMD is attached. The displacement of the tower at the location of the ATMD is therefore given by $\mathbf{w}^{T} \mathbf{u}$, whereby the EOM for the ATMD is given as

$$
m_{a} \ddot{u}_{a}+c_{a} \dot{u}_{a}+k_{a} u_{a}=-m_{a} \mathbf{w}^{T} \ddot{\mathbf{u}}+f_{a} .
$$

Inserting (3) into (2) gives the more convenient representation of the structural EOM

$$
\left(\mathbf{M}+\mathbf{M}_{a}\right) \ddot{\mathbf{u}}+\mathbf{K u}=\mathbf{f}-m_{a} \mathbf{w} \ddot{u}_{a},
$$

where $\mathbf{M}+\mathbf{M}_{a}=\mathbf{M}+\mathbf{w} \mathbf{w}^{T} m_{a}$ is the original mass matrix plus the additional mass of the ATMD. The equation system in (4) together with equation (3) constitutes the EOMs for the wind turbine with an ATMD. By observing the two equations the closed-loop characteristics are described by $-m_{a} \mathbf{w}^{T} \ddot{\mathbf{u}}$ as an input term in $(3)$ and by $-m_{a} \mathbf{w} \ddot{u}_{a}$ as a corresponding output term acting as a force on the wind turbine structure in (4). The active element of the ATMD with control force $f_{a}$ can be used to increase the input force to the external damper system, whereby the output force to the wind turbine is increased as well, so that the overall damping performance can be improved. In the present article the absolute displacement $\mathbf{w}^{T} \mathbf{u}$ of the tower and the relative velocity $\dot{u}_{a}$ of the damper mass are used as sensor signals in the feedback control. Hereby the actuator force is given by

$$
f_{a}=-G_{k} \mathbf{w}^{T} \mathbf{u}-g_{c} c_{a} \dot{u}_{a}
$$

where $G_{k}$ is the gain associated with the absolute displacement of the tower, while $g_{c}$ is the non-dimensional gain associated with feedback of the relative velocity of the damper mass. The introduction of the auxiliary mass of the ATMD provides an additional point of fixture that enables control forces proportional to absolute motion. In (5) the first term acts on the absolute displacement and thus governs the reduction of the structural vibration amplitude via the feedback 
gain $G_{k}$. Although this term is of the same proportional type as in optimal LQR control $[14,15]$ it only requires the top tower displacement $\left(\mathbf{w}^{T} \mathbf{u}\right)$ as input and not the entire dynamic state of the structure. Furthermore, the introduction of pure proportional feedback may result in large actuator forces. Therefore, the second direct velocity feedback term in the control equation (5) represents additional viscous damping with gain $g_{c}$, which introduces a phase-shift in $f_{a}$ that reduces the required actuator force.

The displacement $\mathbf{w}^{T} \mathbf{u}$ of the tower might be derived from high-precision GPS measurements as in $[20,21]$, while the relative velocity of the damper mass $\dot{u}_{a}$ could be derived from the corresponding relative displacement and acceleration signals using a so-called kinematic Kalman filter [22, 23]. Inserting (5) into equations (4) and (3) provides the closed-loop system of equations for the wind turbine and ATMD as

$$
\left[\begin{array}{cc}
\mathbf{M}+\mathbf{M}_{a} & \mathbf{w} m_{a} \\
m_{a} \mathbf{w}^{T} & m_{a}
\end{array}\right]\left[\begin{array}{c}
\ddot{\mathbf{u}} \\
\ddot{u}_{a}
\end{array}\right]+\left[\begin{array}{cc}
\mathbf{0} \mathbf{0}^{T} & \mathbf{0} \\
\mathbf{0}^{T} & \left(1+g_{c}\right) c_{a}
\end{array}\right]\left[\begin{array}{c}
\dot{\mathbf{u}} \\
\dot{u}_{a}
\end{array}\right]+\left[\begin{array}{cc}
\mathbf{K} & \mathbf{0} \\
G_{k} \mathbf{w}^{T} & k_{a}
\end{array}\right]\left[\begin{array}{c}
\mathbf{u} \\
u_{a}
\end{array}\right]=\left[\begin{array}{c}
\mathbf{f} \\
0
\end{array}\right],
$$

where $\mathbf{0}$ is the zero vector. From (6) it is seen that the stiffness matrix is positive definite for any value of $G_{k}$, while for $g_{c}>-1$ the damping matrix is positive semi-definite. Thus, for $g_{c}>-1$ all the eigenfrequencies will have positive imaginary part, and therefore the system will be stable.

2.1. Modal representation of wind turbine structure. An ATMD is usually targeted at one specific mode, which suggests the use of a modal representation for the wind turbine. The displacement of the wind turbine with respect to mode $j$ is described in terms of the mode-shape vector $\mathbf{u}_{j}$ as

$$
\mathbf{u}=\mathbf{u}_{j} r_{j},
$$

where $r_{j}$ is the modal coordinate, whereby the modal mass and stiffness associated with mode $j$ are given by

$$
m_{j}=\mathbf{u}_{j}^{T} \mathbf{M} \mathbf{u}_{j} \quad, \quad k_{j}=\mathbf{u}_{j}^{T} \mathbf{K} \mathbf{u}_{j} .
$$

It is convenient to scale the mode shape vector $\mathbf{u}_{j}$ to unity at the location of the damper, so that $\mathbf{w}^{T} \mathbf{u}_{j}=1$. Introducing the modal representation (7) with this scaling into (6) the two EOMs for the modal coordinate $r_{j}$ and the relative displacement $u_{a}$ of the ATMD can be written as

$$
\left[\begin{array}{cc}
\left(1+\mu_{j}\right) & \mu_{j} \\
1 & 1
\end{array}\right]\left[\begin{array}{c}
\ddot{r}_{j} \\
\ddot{u}_{a}
\end{array}\right]+\left[\begin{array}{cc}
0 & 0 \\
0 & \left(1+g_{c}\right) 2 \zeta_{a} \omega_{a}
\end{array}\right]\left[\begin{array}{c}
\dot{r}_{j} \\
\dot{u}_{a}
\end{array}\right]+\left[\begin{array}{cc}
\omega_{j}^{2} & 0 \\
g_{k} \omega_{j}^{2} / \mu_{j} & \omega_{a}^{2}
\end{array}\right]\left[\begin{array}{c}
r_{j} \\
u_{a}
\end{array}\right]=\left[\begin{array}{c}
f_{j} / m_{j} \\
0
\end{array}\right]
$$

where $f_{j}=\mathbf{u}_{j}^{T} \mathbf{f}$ is the modal load. The equations are characterized by the modal mass ratio $\mu_{j}$, the natural frequency $\omega_{j}$ associated with mode $j$, the frequency $\omega_{a}$ of the ATMD, the damping ratio $\zeta_{a}$ of the ATMD, the non-dimensional modal feedback gain $g_{k}$ associated with the displacement of the tower and the non-dimensional gain parameter $g_{c}$,

$$
\mu_{j}=\frac{m_{a}}{m_{j}}, \quad \omega_{j}^{2}=\frac{k_{j}}{m_{j}}, \quad \omega_{a}^{2}=\frac{k_{a}}{m_{a}}, \quad 2 \zeta_{a}=\frac{c_{a}}{\sqrt{m_{a} k_{a}}}, \quad g_{k}=\frac{G_{k}}{k_{j}} .
$$

Thus, the new displacement proportional gain $g_{k}$ is non-dimensional. Assuming harmonic solutions for the equations in (9) on the form

$$
r_{j}=\bar{r}_{j} \exp (i \omega t), \quad u_{a}=\bar{u}_{a} \exp (i \omega t), \quad f_{j}=\bar{f}_{j} \exp (i \omega t),
$$


with modal amplitudes denoted by a bar and with $\omega>0$ as the forcing frequency, the frequency response of the modal coordinate $r_{j}$ is given by

$$
\frac{\bar{r}_{j}}{r_{j, s t a}}=\frac{\omega_{j}^{2}\left[\omega_{a}^{2}-\omega^{2}+2 i\left(1+g_{c}\right) \zeta_{a} \omega \omega_{a}\right]}{\omega^{4}-\left[\omega_{j}^{2}\left(1-g_{k}\right)+\omega_{a}^{2}\left(1+\mu_{j}\right)\right] \omega^{2}+\omega_{a}^{2} \omega_{j}^{2}+2 i\left(1+g_{c}\right) \zeta_{a} \omega \omega_{a}\left[\omega_{j}^{2}-\omega^{2}\left(1+\mu_{j}\right)\right]},
$$

where $r_{j, s t a}=\bar{f}_{j} / k_{j}$ is the static displacement of mode $j$. The frequency response of the damper mass is given by

$$
\frac{\bar{u}_{a}}{r_{j, s t a}}=\frac{\omega_{j}^{2}\left[\omega^{2}-g_{k} \omega_{j}^{2} \mu_{j}^{-1}\right]}{\omega^{4}-\left[\omega_{j}^{2}\left(1-g_{k}\right)+\omega_{a}^{2}\left(1+\mu_{j}\right)\right] \omega^{2}+\omega_{a}^{2} \omega_{j}^{2}+2 i\left(1+g_{c}\right) \zeta_{a} \omega \omega_{a}\left[\omega_{j}^{2}-\omega^{2}\left(1+\mu_{j}\right)\right]} .
$$

2.2. Optimum frequency tuning. The optimum frequency tuning of the ATMD is now determined with respect to mode $j$. Figure (2) shows a plot of the dynamic amplification of mode $j$ as given by (12) for different values of the modified damping ratio $\left(1+g_{c}\right) \zeta_{a}$. Similar to the passive TMD the ATMD gives rise to two neutral frequencies, denoted as $\omega_{A}$ and $\omega_{B}$, which are located on both sides of the structural frequency $\omega_{j}$ and at which the magnitude of the frequency response is independent of the damping parameter. The optimum frequency tuning of the ATMD is determined by setting the magnitude at these two neutral frequencies equal to each other, equivalent to the optimum frequency tuning of the passive TMD, credited to Den Hartog [24]. The following derivations follow the analysis of Krenk in [25]. First the neutral frequencies $\omega_{A}$ and $\omega_{B}$ are determined by setting the magnitude of (12) equal at the limits $\left(1+g_{c}\right) \zeta_{a}=0$ and $\left(1+g_{c}\right) \zeta_{a} \rightarrow \infty$, which leads to

$$
\left[\omega_{a}^{2}-\omega^{2}\right]\left[\omega_{a} \omega\left(\omega_{j}^{2}-\omega^{2}\left(1+\mu_{j}\right)\right)\right]= \pm \omega_{a} \omega\left[\omega^{4}-\left(\omega_{j}^{2}\left(1-g_{k}\right)+\omega_{a}^{2}\left(1+\mu_{j}\right)\right) \omega^{2}+\omega_{a}^{2} \omega_{j}^{2}\right] .
$$

Use of the plus sign leads to the roots $\omega^{2}=0$ and $\omega^{2}=-\omega_{j}^{2} g_{k} / \mu_{j}$, while use of the minus sign gives the following quadratic equation in $\omega^{2}$

$$
\left(2+\mu_{j}\right) \frac{\omega^{2}}{\omega_{j}^{2}} \frac{\omega^{2}}{\omega_{a}^{2}}-2\left[\frac{\omega^{2}}{\omega_{a}^{2}}\left(1-g_{k} / 2\right)+\frac{\omega^{2}}{\omega_{j}^{2}}\left(1+\mu_{j}\right)\right]+2=0 .
$$

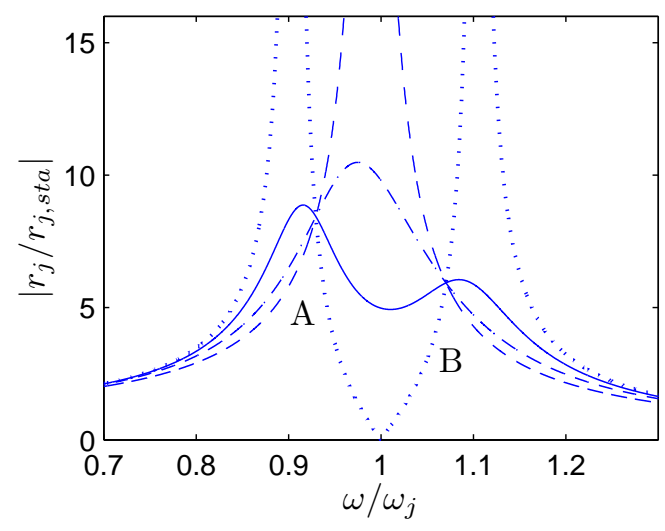

Figure 2. Dynamic amplification for $\mu=-g_{k}=0.02, \omega_{a}=\omega_{j}$ and $\zeta_{a}\left(1+g_{c}\right)=$ $0.0(\cdot), 0.1(-), 0.2(-\cdot-), \infty(--)$ 
The roots of this equation are $\omega_{A}^{2}$ and $\omega_{B}^{2}$. The roots are however only needed in the form of their sum, which can be determined via the ratio of the coefficients to the linear and quadratic terms,

$$
\left(\frac{\omega_{A}}{\omega_{0}}\right)^{2}+\left(\frac{\omega_{B}}{\omega_{0}}\right)^{2}=\frac{2}{2+\mu_{j}}\left[\left(1-g_{k} / 2\right)+\frac{\omega^{2}}{\omega_{a}^{2}}\left(1+\mu_{j}\right)\right] .
$$

Next the magnitude at the two neutral frequencies are set equal to each other. Evaluating (12) at the limit $\left(1-g_{c}\right) \zeta_{a} \rightarrow \infty$ then gives the magnitude at the neutral frequencies

$$
\frac{\bar{r}_{j}}{\bar{r}_{j, s t a}}=\frac{1}{1-\left(1+\mu_{j}\right) \frac{\omega^{2}}{\omega_{j}^{2}}} .
$$

At the lower frequency $\omega_{A}$ the response of mode $j$ is in phase with the external load, while at the higher frequency $\omega_{B}$ the response is in opposite phase. As demonstrated in [25] equal magnitude of the response at the two frequencies results in

$$
\left(\frac{\omega_{A}}{\omega_{0}}\right)^{2}+\left(\frac{\omega_{B}}{\omega_{0}}\right)^{2}=-\frac{2}{1+\mu_{j}},
$$

and combining equation (16) and equation (18) then gives the optimum frequency tuning of the ATMD as

$$
\frac{\omega_{a}}{\omega_{0}}=\frac{1}{\sqrt{2}} \frac{\sqrt{2+g_{k}+g_{k} \mu}}{1+\mu_{j}} .
$$

The optimum frequency tuning is seen to depend both on the mass ratio $\mu_{j}$ and the gain parameter $g_{k}$. Furthermore, $g_{k}=-2 /\left(1+\mu_{j}\right)$ is seen to correspond to a design limit, since it leads to the frequency tuning $\omega_{a}=0$. For $g_{k}=0$ the optimum tuning of the passive TMD is recovered.

By substitution of the the frequency tuning (19) into the quadratic equation in (15) the roots are determined as

$$
(1+\mu) \frac{\omega_{A, B}^{2}}{\omega_{0}^{2}}=1 \pm \sqrt{\frac{\mu-g_{k}-g_{k} \mu}{2+\mu}} .
$$

The equal dynamic amplification at the two neutral frequencies $\omega_{A, B}$ is denoted as $A_{\max }$. It is determined by substituting (20) into (17), which gives

$$
A_{\max }=\sqrt{\frac{2+\mu}{\mu-g_{k}-g_{k} \mu}} .
$$

When compared to the dynamic amplification of the passive TMD it is seen that the ATMD offers further reduction in the dynamic amplification by an appropriate choice of $g_{k}<0$, while in the limit $g_{k}=0$ the dynamic amplification of the mechanical TMD is recovered. The design limit $g_{k}=-2 /\left(1+\mu_{j}\right)$ from $(19)$ is seen to correspond to $A_{\max }=1$, i.e. no dynamic amplification.

2.3. Optimum damper tuning. With the optimum frequency tuning in (19) the dynamic amplification at the two neutral frequencies is equal, and damping of the ATMD should therefore be scaled, so that a flat plateau between the two neutral frequencies is obtained. In [25] the optimum damper tuning of the passive TMD is determined by selecting a suitable frequency in between the two neutral frequencies and setting the dynamic amplification equal at the three frequencies, whereby a fairly flat plateau is obtained. The same approach is applied in the present case for the ATMD. The dynamic amplification at the frequency $\omega_{\infty}=\sqrt{k_{j} /\left(m_{j}+m_{a}\right)}$, which corresponds to the natural frequency of the structure when locking the damper, is thus equated to the dynamic 
amplification at the two neutral frequencies $\omega_{A, B}$. The frequency response at $\omega_{\infty}$ follows from (12) as

$$
\left[\frac{\bar{r}_{j}}{r_{j, s t a}}\right]_{\omega_{\infty}}=\frac{\left(\mu_{j}-g_{k}-g_{k} \mu_{j}\right)-2 i \zeta_{a} \sqrt{1+g_{k} / 2+g_{k} / 2 \mu_{j}} \sqrt{1+\mu_{j}}\left(1+g_{c}\right)}{\mu_{j}-g_{k}-g_{k} \mu_{j}},
$$

whereby the magnitude is determined as the square root of

$$
\left|\frac{\bar{r}_{j}}{r_{j, s t a}}\right|_{\omega_{\infty}}^{2}=\frac{\left(\mu_{j}-g_{k}-g_{k} \mu_{j}\right)^{2}+4 \zeta_{a}^{2}\left(1+g_{k} / 2+g_{k} / 2 \mu_{j}\right)\left(1+\mu_{j}\right)\left(1+g_{c}\right)^{2}}{\left(\mu_{j}-g_{k}-g_{k} \mu_{j}\right)^{2}} .
$$

When equating the square of the dynamic amplification at the three frequencies $\omega_{\infty}$ and $\omega_{A, B}$ the following relation is obtained

$$
\frac{2+\mu}{\mu-g_{k}-g_{k} \mu}=\frac{\left(\mu_{j}-g_{k}-g_{k} \mu_{j}\right)^{2}+4 \zeta_{a}^{2}\left(1+g_{k} / 2+g_{k} / 2 \mu_{j}\right)\left(1+\mu_{j}\right)\left(1+g_{c}\right)^{2}}{\left(\mu_{j}-g_{k}-g_{k} \mu_{j}\right)^{2}}
$$

which determines the optimum damping ratio as

$$
\left(1+g_{c}\right) \zeta_{a}=\sqrt{\frac{1}{2} \frac{\mu_{j}-g_{k}\left(1+\mu_{j}\right)\left(1+g_{k} / 8\left(1+\mu_{j}\right)\right)}{1+\mu_{j}+g_{k} / 2\left(1+\mu_{j}\right)^{2}}} .
$$

For most feasible designs with $\mu_{j}<<1$ and $\left|g_{k}\right|<<1$ this expression for the optimum damper tuning can be readily approximated to give

$$
\left(1+g_{c}\right) \zeta_{a} \simeq \sqrt{\frac{1}{2} \frac{\mu_{j}-g_{k}}{1+\mu_{j}+g_{k} / 2}} .
$$

Together with the expression in (19) the expression above in (26) gives the optimum frequency and damper tuning for the ATMD, while for $g_{k}=g_{c}=0$ the optimum frequency and damper tuning of the passive TMD is recovered [25]. Comparison of the performance of the ATMD with that of the passive TMD in figure 3 shows that with active control the response of mode $j$ can be reduced without increasing the damper mass, while the maximum damper mass amplification is only slightly increased. The displacement $u_{a}$ is however increased significantly for frequencies below the structural frequency. Thus, the ATMD can provide further amplitude reduction compared to the passive TMD, at the expense of a larger damper mass displacement.

(a)

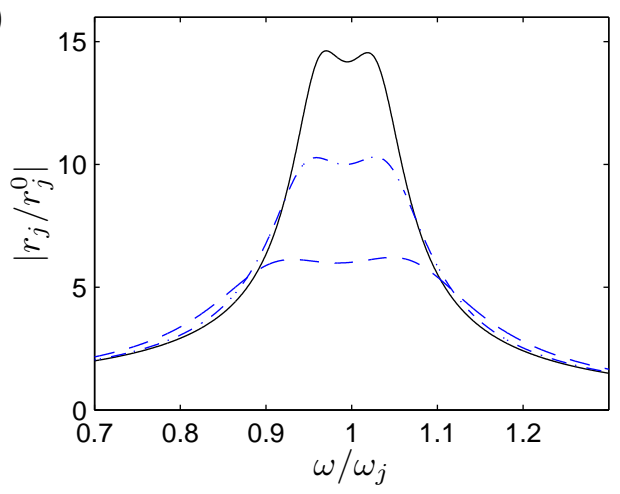

(b)

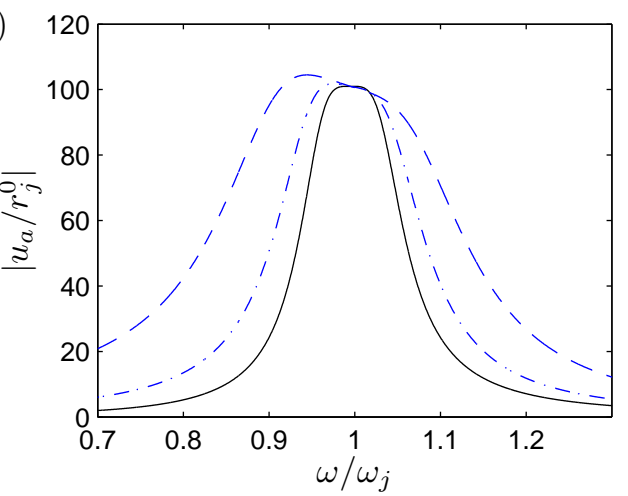

Figure 3. Dynamic amplification of mode $j$ (a) and the damper mass (b) for $\mu=0.01$ and $A_{\max }=14.17\left(g_{k}=0\right)(-), A_{\max }=10(-\cdot-)$ and $A_{\max }=6(--)$ 


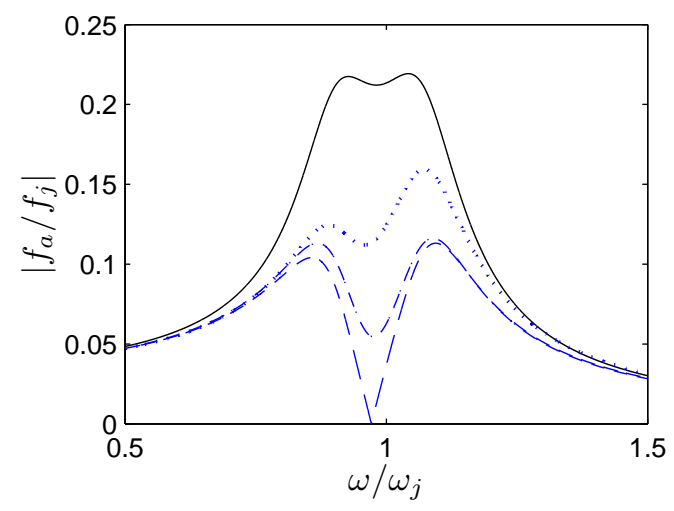

Figure 4. Magnitude of the the normalized control force, for $g_{c}=0(-), g_{c}=$ $g_{c}^{\text {opt }} / 2(-\cdot-), g_{c}=g_{c}^{o p t}(--)$ and $g_{c} \rightarrow \infty(\cdot)$, and for $\mu=0.02$ and $A_{\max }=6$.

2.4. Tuning for reduction of control force. Proper tuning of the gain parameter $g_{c}$ is determined by studying the active control force of the ATMD as given by equation (5). The active control force has two terms, the first which is proportional to $G_{k}=g_{k} k_{j}$, and the second which is proportional to $g_{c}$. Close to the structural frequency $\omega_{j}$ the second term in (5) proportional to $g_{c}$ can be calibrated to compensate for the additional contribution to the control force $f_{a}$ from the displacement proportional first term with gain $g_{k}$. By assuming harmonic solutions as in (11) the frequency response of the control force is determined as

$$
\left[\begin{array}{c}
\bar{f}_{a} \\
\bar{f}_{j}
\end{array}\right]=-\frac{g_{k} \omega_{j}^{2}\left[\omega_{a}^{2}-\omega^{2}\right]+2 i \zeta_{a} \omega_{a} \omega \omega_{j}^{2}\left[g_{k}+g_{c} \omega_{a}^{2} / \omega_{j}^{2} \mu_{j}\right]}{\omega^{4}-\left[\omega_{j}^{2}\left(1-g_{k}\right)+\omega_{a}^{2}\left(1+\mu_{j}\right)\right] \omega^{2}+\omega_{a}^{2} \omega_{j}^{2}+2 i \zeta_{a} \omega \omega_{a}\left(1+g_{c}\right)\left[\omega_{j}^{2}-\omega^{2}\left(1+\mu_{j}\right)\right]} .
$$

When closely studying the frequency response in (27) it is found that by choosing $g_{c}$ according to

$$
g_{c}=-\frac{g_{k}}{\mu_{a}} \frac{\omega_{0}^{2}}{\omega^{2}}
$$

the control force amplitude is completely canceled at the frequency $\omega=\omega_{a}$. Figure (4) shows the magnitude of the frequency response of the control force for different values of $g_{c}$. The curve $(--)$ with the tuning according to (28) is seen to have a minimum at the frequency $\omega_{a}$, where the control force is zero. Compared to the case $g_{c}=0(-)$, this gives a significant reduction in the control force around $\omega_{j}$, while for $\omega \rightarrow 0$ and $\omega \rightarrow \infty$ the difference for different values for $g_{c}$ becomes insignificant. Therefore, the tuning in (28) is in the following chosen as the optimum tuning of $g_{c}$, since it effectively reduces the control effort around the resonance frequency.

\section{DAMPING OF OFFSHORE WIND TURBINE TOWER VIBRATIONS}

The performance of the ATMD with respect to damping of offshore wind turbine tower vibrations is now investigated by use of time simulations in HAWC2 [19]. The aeroelastic code HAWC2 (Horzontal Axis Wind Turbine simulation Code 2nd generation) has been developed at DTU Wind Energy (Technical University of Denmark) for realistic time simulations of wind turbines and it is used in connection with research projects as well as industrial applications. The code includes a multi body formulation for modeling of nonlinear structural dynamics, a Stig Øye model for aerodynamic 
TABLE 1. Structural properties of the OC3 reference wind turbine

\begin{tabular}{ll}
\hline Description & Value \\
\hline Hub height [m] & 90 \\
Tower-top height above MSL [m] & 87.6 \\
Tower-base height above MSL [m] & 10 \\
Water depth from MSL [m] & 20 \\
Penetration depth of monopile [m] & 36 \\
Tower mass [ton] & 237 \\
Mass of Nacelle+Rotor+Blades [ton] & 351 \\
Overall integrated mass [ton] & 1216 \\
\hline
\end{tabular}

modeling of the blades, a hydrodynamic model for modeling of wave loading and added mass, and a nonlinear spring model for modeling of stiffness and damping of the flexible foundation provided by the soil. The wind turbine model used for the simulations is from the OC3 (Offshore Code Comparison Collaboration) project Phase 2 [26], and the numerical model has been downloaded from www.hawc2. dk. The wind turbine is a slightly modified version of the 5 MW NREL reference wind turbine [27], which is installed on a monopile with a flexible soil foundation at $20 \mathrm{~m}$ of water depth. The tower is approximately $78 \mathrm{~m}$ tall and tapered from bottom to top, while the monopile has a constant thickness and diameter. Some of the main structural characteristics of the wind turbine are summarized in table 1 . In order to reduce vibrations of the two lowest critical tower modes, one ATMD is installed in the rotor direction and one ATMD is installed in the sideways direction. Implementation of the external damper systems into HAWC2 is done using an external dynamic link library (dll), as described in more detail for an advanced dynamic gear model in [28]. The ATMDs are connected to the first node of the tower top at $87.6 \mathrm{~m}$ above Mean Sea Level (MSL), where the modal amplitude of the two tower modes is expected to be large.

3.1. Modal properties of critical tower modes. Optimum tuning of the ATMDs according to the expressions in (19), (21), (25) and (28) requires information about the critical modes of the wind turbine, including information about the natural frequency, modal mass and modal stiffness. In order to assess the modal properties, two simple decay analyzes are conducted. In the decay analysis the wind turbine without ATMD is assumed at standstill, and the shaft and pitch bearings are thus restrained. The influence of the wind is omitted and the wave height is set to zero, whereby aerodynamic damping is excluded, while the contribution from added mass

TABLE 2. Modal properties of two lowest tower modes

\begin{tabular}{lcccc}
\hline & $\omega_{0} /(2 \pi)[\mathrm{Hz}]$ & $\zeta[\%]$ & $m_{j}[$ ton $]$ & $k_{j}[\mathrm{kN} / \mathrm{m}]$ \\
Side-to-side & 0.2379 & 1.14 & 450 & 1006 \\
Fore-aft & 0.2390 & 1.15 & 440 & 990 \\
Average & 0.2385 & 1.15 & 445 & 999 \\
\hline
\end{tabular}

10 
(a)

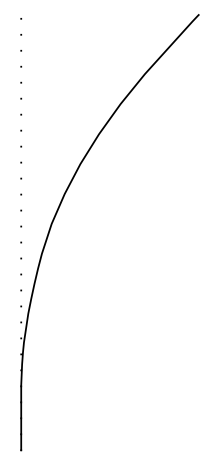

(b)

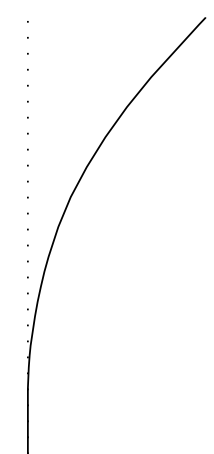

Figure 5. Illustration of the (a) side-side mode and (b) fore-aft mode

is maintained. Instead of the load from wind and waves the turbine is loaded by a single-step load at the top of the tower, and the natural frequency, damping ratio and mode shape is estimated from the free vibrations of the wind turbine. The damping ratio of the two modes is found to be approximately $\zeta=0.0020$ with soil damping representing approximately one fifth (0.0004), while the remaining amount (0.0016) comes from proportional damping in the numerical model. In order to increase this to a more realistic value soil damping is simply calibrated to get a total damping ratio in both modes slightly larger than 0.01 , which corresponds to experimentally predicted values in the literature $[2,29,30]$. The resulting values for the natural frequencies and damping ratios are presented in table 2 . The mode shapes of the two tower modes are estimated by taking the fourier transform of the decay signal for each node, and comparing amplitudes of the peaks at the tower frequency. The mode shapes are depicted in figure 5. In order to also estimate the modal mass and stiffness of the two modes, the mass matrix of the wind turbine is reproduced by lumping the mass of each element in the tower and monopile and the mass and inertia of the nacelle, generator, hub and blades. The modal mass is subsequently computed by using the mode shape determined from the decay analysis, and next the modal stiffness is determined from the modal mass and the natural frequency. The estimated values for the modal mass and modal stiffness are also given in table 2. As seen from the table the modal properties of the two tower modes are almost the same and it therefore seems reasonable for the two ATMDs acting in the rotor direction and lateral to the rotor direction to be tuned according to the average modal parameters as given in table 2. The optimum ATMD parameter values computed from these average modal properties for a mass ratio of $\mu=0.01$ are summarized in table 3. These ATMD parameter values are used in the simulations in the following sections.

3.2. Frequency response. In order to estimate the frequency response of the wind turbine a series of time responses are computed. As for the estimation of the modal properties the wind turbine is again assumed to be at stand still, the wind loading is removed and the wave height is set to zero. In each time simulation the wind turbine is instead loaded at MSL by a time 
varying harmonic force, either in the sideways direction or in the direction normal to the rotor plane. The frequency of the harmonic loading is increased slightly for each simulation, and after the initial transient response the corresponding amplitude of the steady state harmonic response is determined. The estimated dynamic amplification for the wind turbine tower top and damper displacement in the two (sideways and rotor) directions are shown in figure 6. As expected the tower top response curves is seen to be almost identical for the two directions. Assuming that the wind turbine is vibrating primarily at resonance in a single vibration mode the dynamic amplification is inversely proportional to the damping ratio via the factor $1 /(2 \zeta)$. In the case without a mass damper the amplitude at resonance is approximately 44.6, which corresponds to a damping ratio of $\zeta=0.0115$, as also estimated by the modal damping ratio determined in Section 3.1. When comparing the response of the full wind turbine with the corresponding frequency response curves for the idealized single-DOF system in figure 3 , the response of the wind turbine top in figure 6 is seen to have a slightly different shape and thereby frequency dependence. This discrepancy is mainly due to the modal approximation of the wind turbine leading to the single-DOF system and because of the small off-tuning of the damper parameters caused by using the average modal properties for the two tower modes. The amplitude of the response is also seen to be even smaller than predicted for the single-DOF system. This is due to the inherent damping $\zeta=0.0115$ in the wind turbine model, which is not included in the results for the single-DOF structure. In [25] it has been demonstrated that for the passive TMD the tuning in (19) corresponds to dividing the added damping by the mass damper equally between the two vibration modes associated with the targeted vibration form of the structure. Assuming that the tower top response at the two neutral frequencies is approximately given by the amplitude factor $1 /(2 \zeta)$ the corresponding damping ratio for the passive case is estimated to be $\zeta=0.0467$, which corresponds well with the sum of half of the added damping 0.0352 from table 3 and the inherent damping 0.0115. Following the same line of analysis the damping ratio for the case with $A_{\max }=10$ is estimated to $\zeta=0.0613$, while for $A_{\max }=6$ the estimation gives $\zeta=0.0948$. This again corresponds well with the inhenrent damping of 0.0115 plus half of the added damping 0.0499 and 0.0837 , respectively. Thus, structural damping and damping from the ATMD appear to be additive during steady-state harmonic motion. Also the relative displacement of the damper mass decreases compared to the single-DOF case, although this reduction is more pronounced for the passive tuned mass damper than for the ATMD.

TABLE 3. ATMD properties for $\mu=0.01$

\begin{tabular}{lccccccc}
\hline$A_{\max }$ & $\omega_{a} /(2 \pi)[\mathrm{Hz}]$ & $\zeta_{a}\left(1+g_{c}\right)[\%]$ & $m_{a}[\mathrm{~kg}]$ & $k_{a}[\mathrm{~N} / \mathrm{m}]$ & $c_{a}[\mathrm{~N}-\mathrm{s} / \mathrm{m}]$ & $g_{k}$ & $g_{c}$ \\
14.18 & 0.2361 & 7.04 & 4450 & 9796 & 929 & 0.000 & 0.00 \\
10 & 0.2355 & 9.98 & 4450 & 9747 & 649 & -0.010 & 1.03 \\
6 & 0.2334 & 16.75 & 4450 & 9572 & 381 & -0.045 & 4.74 \\
\hline
\end{tabular}

12 
(a)

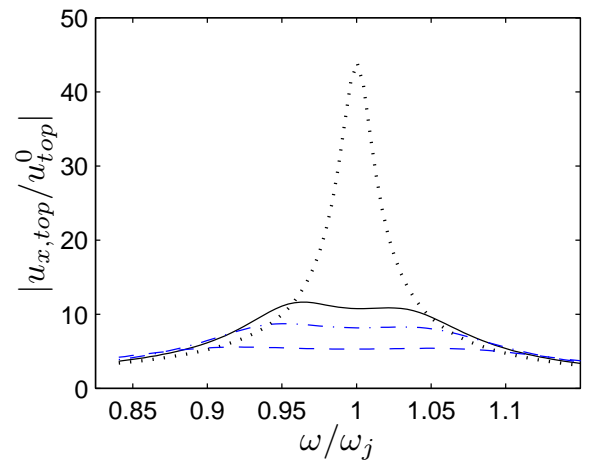

(c)

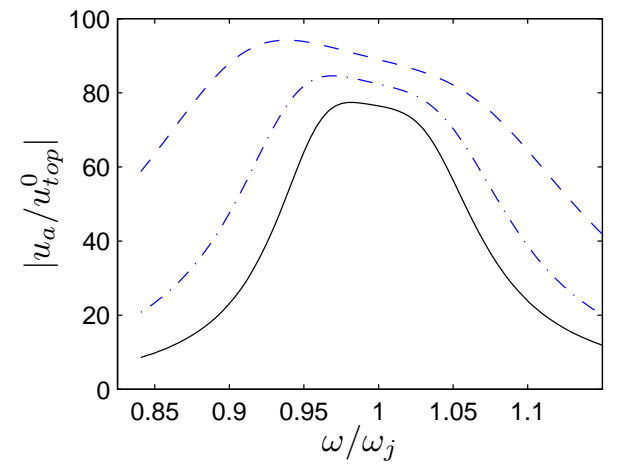

(b)

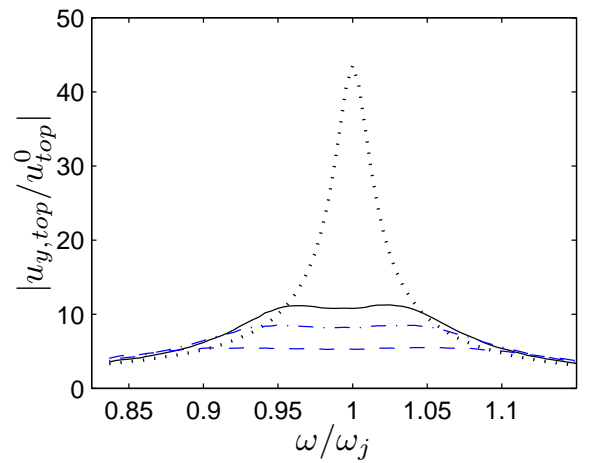

(d)

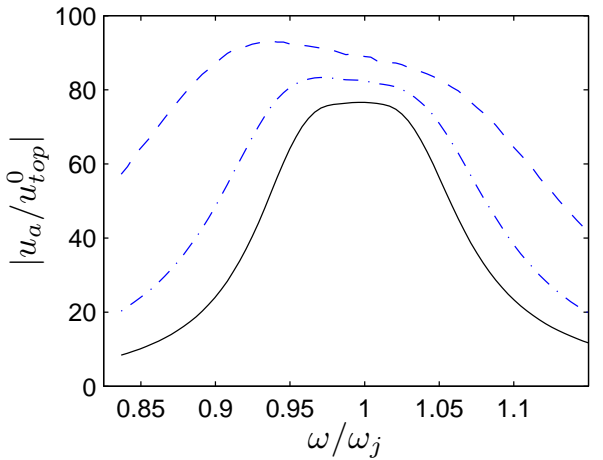

FiguRE 6. Dynamic amplification of tower top for (a) sideways - and (b) rotor direction and of damper for (c) sideways - and (d) rotor direction for $\mu=0(\cdot)$ and for $\mu=0.01$ and $\left.A_{\max }=14.17\left(g_{k}=g_{c}=0\right)(-), A_{\max }=10(-\cdot-)\right)$ and $A_{\max }=6(--)$

3.3. Time domain simulation. As demonstrated in the previous section the ATMD is very effective in reducing the steady state response of the wind turbine around resonance. To investigate the the ability of the ATMD to mitigate the transient vibrations following the impact of large waves, the wind turbine with the ATMDs installed is now analyzed through time domain response simulations. The wind turbine is loaded by a mean wind speed of $8 \mathrm{~m} / \mathrm{s}$, with zero turbulence intensity and a constant shear profile with a power law exponent of 0.14 according to [19]. The aerodynamic drag on the tower and nacelle is also included. In addition to the wind load the wind turbine is also loaded by a wave train in a direction of $45^{\circ}$ relative to the rotor direction. Hereby both the fore-aft mode (rotor direction) and side-side mode (sideways direction) are excited by the misaligned wave loading. The wave train is introduced as three consecutive sine waves at MSL with $10 \mathrm{~s}$ wave period. After the wave train has passed the wave loading is set to zero. Furthermore, the wave load is applied sufficiently long time after simulation startup, so that the initial transients from the wind loading can be neglected.

Figure 7 shows the response of the tower top. As expected the vibrations in the rotor direction (fore-aft mode) has a larger decay rate than the vibrations in the sideways direction (side-side mode). This is due to the large additional aerodynamic damping introduced in the rotor direction. The vibrations in the rotor direction also have a non-zero mean, due to the corresponding mean wind pressure, applying a constant load on the wind turbine. The damping in the two directions 
(modes) is estimated by an exponential fit to the vibration peaks of the free response of the wind turbine. A close-up of the free vibration part of the dynamic response is shown in figure $7(\mathrm{c}, \mathrm{d})$. The solid curves represent the case without mass damper $(\mu=0)$, while the dash-dotted and dashed curves represent the ATMD with $\mu=0.01$ and $A_{\max }=14.17$ (TMD) and 6 (ATMD), respectively. For the case without mass damper (solid curves) the critical damping ratio of the vibrations in the rotor direction (fore-aft) is estimated to $\zeta_{f a}=0.1113$, which is significantly larger compared to standstill because of the aerodynamic damping. By the same procedure the damping ratio of the sideways vibrations (side-side mode) is estimated to $\zeta_{s s}=0.0124$, which is slightly higher than at standstill. This small increase, compared to 0.0115 at standstill, is mainly due to modal interaction between the vibrations in the rotor (fore-aft) and sideways (side-side) directions and the small aerodynamic damping also present in the sideways direction.

When including a TMD (dash-dotted curves) or an ATMD (dashed curves) it is seen in figure $7(\mathrm{c}, \mathrm{d})$ that the decay rate for the free tower vibrations increases. However, the free vibration decay is no longer described by an exponential function. Instead a clear beating phenomenon is observed, where the amplitude of the regular vibrations are modulated by a slower vibrational behavior. This illustrates the interaction between the structure and mass damper, which obviously complicates the estimation of the damping ratio by an exponential fit procedure. However, by a suitable mean value through the decay curves the damping ratios for the TMD are estimated as $\zeta_{s s}^{T M D}=0.0476$ (sideways direction) and $\zeta_{f a}^{T M D}=0.1465$ (rotor direction), while for the ATMD the corresponding damping ratios increase to $\zeta_{s s}^{A T M D}=0.0961$ and $\zeta_{f a}^{A T M D}=0.1950$. Again this corresponds well with the inherent damping $\zeta_{s s}=0.0124$ (sideways direction) and $\zeta_{f a}=0.1113$ (rotor direction) plus half the added damping according to table 3.

In figure 8 the relative displacement of the damper mass and the force exerted by the damper on the tower are shown for the TMD and ATMD. As expected the vibrations of the ATMD are larger than the vibrations of the passive TMD. However, this happens primarily during the initial part when the three waves pass the turbine. Therefore, it might partly be explained by the reported increase in vibrational amplitude in figure $6(\mathrm{c}, \mathrm{d})$ and partly by the contribution from the tower top displacement $u_{t o p}=\mathbf{w}^{T} \mathbf{u}$ in figure $7(\mathrm{a}, \mathrm{b})$, which is amplified by $G_{k}$ in the control equation (5). To avoid the latter, a high-pass filter could be used to filter the tower displacement signal before it is fed back to the actuator via (5). During the beginning of the free decay in figure 8 the amplitude of the ATMD response is still larger compared to the TMD response. However, after only a few periods the magnitude of the ATMD response is smaller than the response of the TMD, mainly because the vibrations of the tower are mitigated more by the ATMD. In figure 8(c,d) for total damper force a similar trend is observed. During the initial loading period and the initial phase of the free response the force is larger for the ATMD than for the TMD, while after only a few periods of the free response the force exerted by the ATMD is reduced below the force of the TMD. 

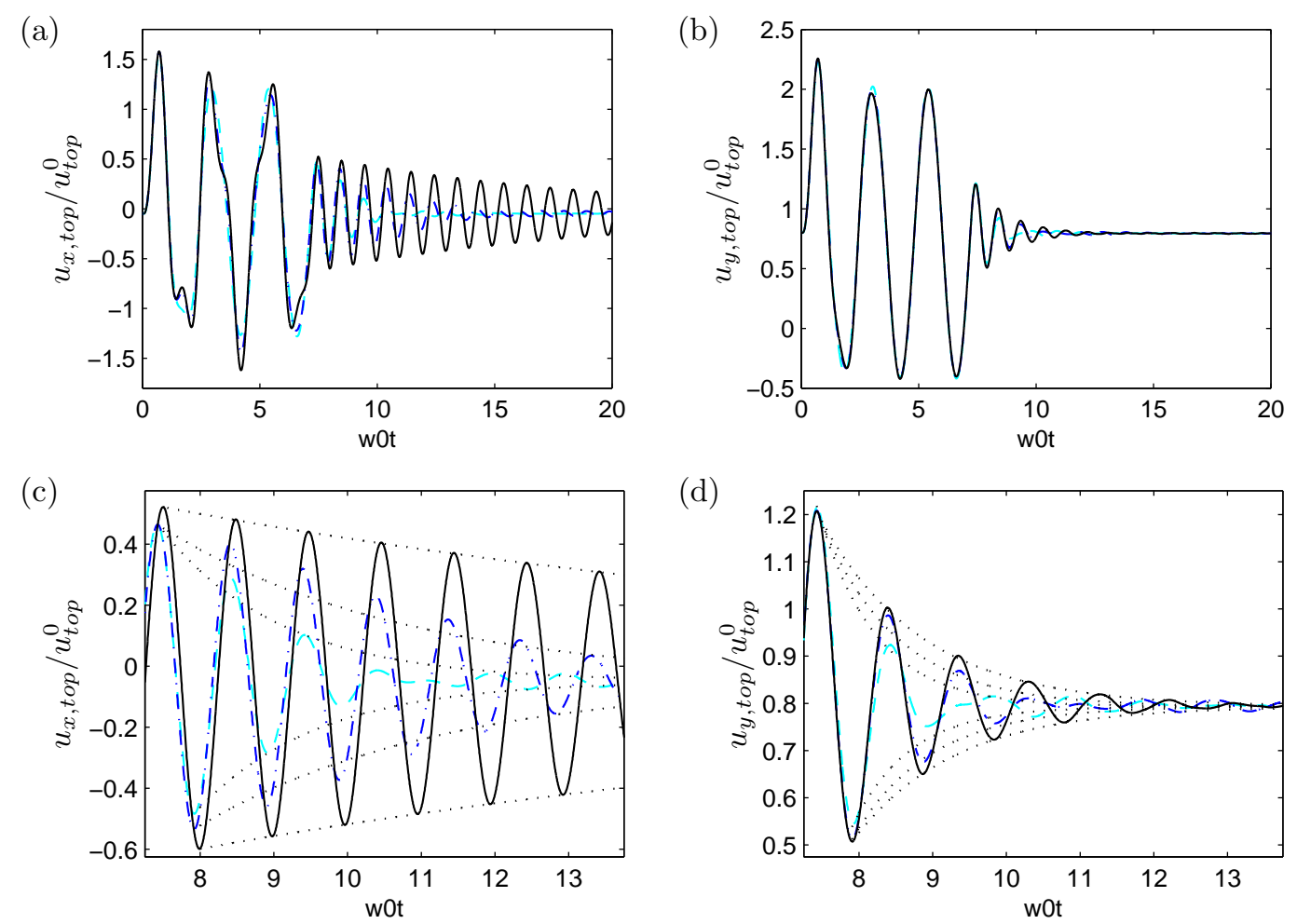

Figure 7. Time response of tower top for (a) and (c) sideways - and (b) and (d) rotor direction for $\mu=0(-)$ and for $\mu=0.01$ and $A_{\max }=14.17\left(g_{k}=g_{c}=0\right)$ $(-\cdot-)$ and $A_{\max }=6(--)$

\section{Conclusion}

Dynamic vibration absorber concepts traditionally used for damping of tower vibrations in fixed offshore wind turbines is limited in effectiveness by the size of the damper mass. Since additional mass at the top of the tower is highly undesirable, the present paper considers an Active Tuned Mass Damper (ATMD) for damping of tower vibrations. Feedback of the tower top displacement and of the relative velocity of the damper mass is introduced to control the active element of the ATMD. By analogy to the optimum tuning of the passive TMD [25], a frequency tuning is introduced for a simple one degree of freedom system that sets the amplitude at the two "neutral frequencies" equal to each other. Furthermore, a damper tuning is introduced that produces a flat curve for the response amplitude around the tower resonance frequency in the frequency domain. And furthermore it minimizes the control effort required to operate the ATMD. The ATMD is found to provide a significant decrease in the frequency response amplitude compared to the passive TMD without an increase in damper mass and the added damping is found to be almost additive to the inherent damping from structural, soil and aerodynamic damping. A time response analysis demonstrates that the ATMD is also superior in reducing the transient, when compared to the passive TMD, and the damping ratio as predicted by the frequency response analysis seems to be almost attainable. The reduced mass, however, comes at the compromise of an increased damper 
(a)

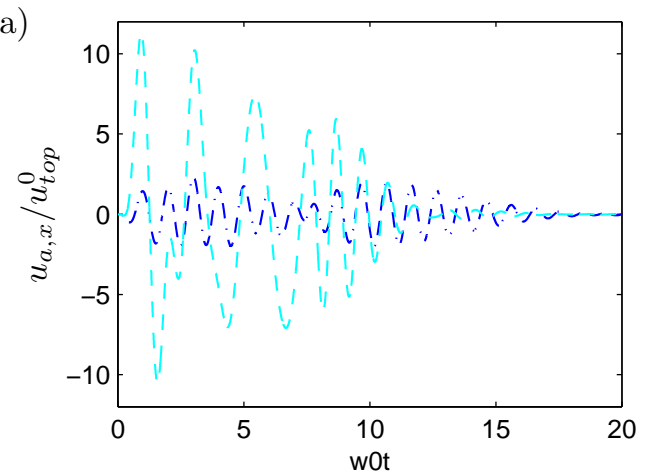

(c)

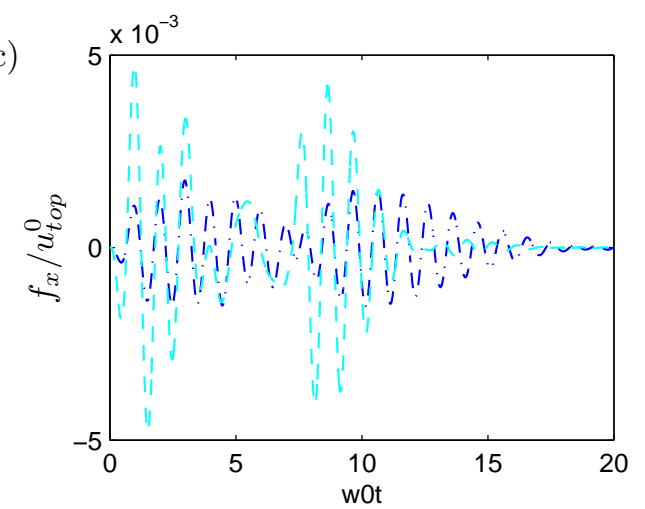

(b)

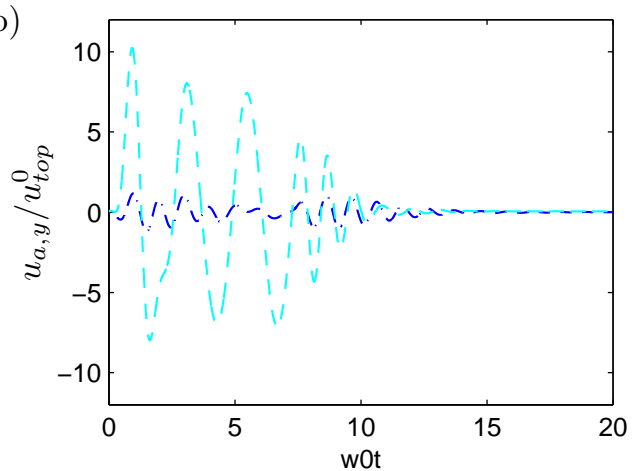

(d)

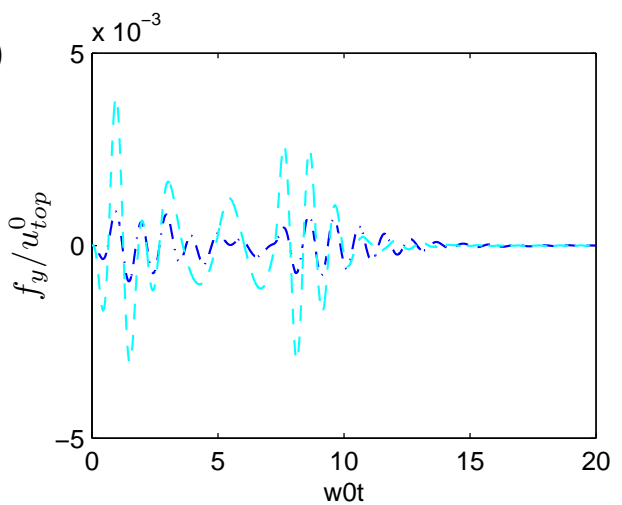

FiguRE 8. Relative displacement of damper mass for (a) sideways damper and (b) rotor direction damper and force exerted by damper for (a) sideways damper and (b) rotor direction damper for $\mu=0.01$ and $A_{\max }=14.17\left(g_{k}=g_{c}=0\right)(-$ $\cdot-)$ and $A_{\max }=6(--)$

mass displacement, which constitutes a limiting factor in the design and feasibility of the proposed ATMD. Especially at frequencies below the tower frequency, the vibrations of the damper mass are increased compared to the passive TMD. This should be compensated by for example introducing a high pass filter to limit contributions from the tower top displacement at quasi-static frequencies below the natural frequencies of the two tower modes.

Acknowledgments. This work has been supported by the Danish Energy Agency and Vestas Wind Systems A/S under the EUDP project 'Monopile cost reduction and demonstration by joint applied research'.

\section{REFERENCES}

[1] NJ Tarp-Johansen, L Andersen, ED Christensen, C Mørch, S Frandsen, and B Kallesøe. Comparing sources of damping of cross-wind motion. In Proceedings of the European Wind Energy Conference \& Exhibition, Stockholm, Sweden, 2009.

[2] M Damgaard, LB Ibsen, LV Andersen, and JKF Andersen. Cross-wind modal properties of offshore wind turbines identified by full scale testing. Journal of Wind Engineering and Industrial Aerodynamics, 116:94-108, 2013. 
[3] C Tibaldi, T Kim, TJ Larsen, F Rasmussen, R de Rocca Serra, and F Sanz. An investigation on wind turbine resonant vibrations. Wind Energy, 2015.

[4] European Wind Energy Association. Report: The european offshore wind industry - key trends and statistics 2013. Technical report, 2014.

[5] S Colwell and B Basu. Tuned liquid column dampers in offshore wind turbines for structural control. Engineering Structures, 31(2):358-368, 2009.

[6] MA Lackner and MA Rotea. Passive structural control of offshore wind turbines. Wind Energy, 14:373-388, 2011.

[7] Gordon M Stewart and Matthew A Lackner. The impact of passive tuned mass dampers and wind-wave misalignment on offshore wind turbine loads. Engineering Structures, 73:54-61, 2014.

[8] Francesco Ricciardelli, A David Pizzimenti, and Massimiliano Mattei. Passive and active mass damper control of the response of tall buildings to wind gustiness. Engineering structures, 25(9):1199-1209, 2003.

[9] Seshasayee Ankireddi and Henry T Y. Yang. Simple atmd control methodology for tall buildings subject to wind loads. Journal of Structural Engineering, 122(1):83-91, 1996.

[10] Yoshiki Ikeda. Active and semi-active vibration control of buildings in japan - practical applications and verification. Structural Control and Health Monitoring, 16(7-8):703-723, 2009.

[11] James CH Chang and Tsu T Soong. Structural control using active tuned mass dampers. Journal of the Engineering Mechanics Division, 106(6):1091-1098, 1980.

[12] CC Chang and Henry TY Yang. Control of buildings using active tuned mass dampers. Journal of engineering mechanics, 121(3):355-366, 1995.

[13] Isao Nishimura, Takuji Kobori, Mitsuo Sakamoto, Norihide Koshika, Katsuyasu Sasaki, and Satoshi Ohrui. Active tuned mass damper. Smart Materials and Structures, 1(4):306, 1992.

[14] B Fitzgerald, B Basu, and SRK Nielsen. Passive structural control of offshore wind turbines. Structural Control and Health Monitoring, 20:1377-1396, 2013.

[15] B Fitzgerald and B Basu. Passive structural control of offshore wind turbines. Engineering Structures, 111:131-151, 2016.

[16] MA Lackner and MA Rotea. Structural control of floating wind turbines. Mechatronics, 21:704-719, 2011.

[17] H Namik, MA Rotea, and MA Lackner. Active structural control with actuator dynamics on a floating wind turbine. In Proceedings of the 51st AIAA Aerospace Sciences Meeting including the New Horizons Forum and Aerospace Exposition 2013, Grapevine, Texas, USA, 2013.

[18] M Rahman, ZC Ong, WT Chong, S Julai, and SY Khoo. Performance enhancement of wind turbine systems with vibration control: A review. Renewable and Sustainable Energy Reviews, 51:43-54, 2015.

[19] Torben J Larsen and Anders Melchior Hansen. How 2 HAWC2, the user's manual. Ris $\varnothing$ National Laboratory, 2007. 
[20] T Kijewski-Correa, A Kareem, and M Kochly. Experimental verification and full-scale deployment of global positioning systems to monitor the dynamic response of tall buildings. Journal of Structural Engineering, 132(8):1242-1253, 2006.

[21] Hyo Seon Park, Hong Gyoo Sohn, Ill Soo Kim, and Jae Hwan Park. Application of gps to monitoring of wind-induced responses of high-rise buildings. Structural Design of Tall and Special Buildings, 17(1):117, 2008.

[22] Charles Henry Boston. Mitigation of stay cable vibrations with magnetorheological dampers. PhD thesis, Diss., Eidgenössische Technische Hochschule ETH Zürich, 2010.

[23] Subrata Bhowmik. Modelling and control of Magnetorheological Damper - Real-time implementation and experimental verification. PhD thesis, Technical University of Denmark, 2012.

[24] J.P. Den Hartog. Mechanical vibrations, 1956. (Reprinted by Dover, New York, 1985).

[25] S Krenk. Frequency analysis of the tuned mass damper. Journal of applied mechanics, 72(6):936-942, 2005.

[26] J Jonkman and W Musial. Offshore code comparison collaboration (OC3) for IEA task 23 offshore wind technology and deployment. Technical report, National Renewable Energy Laboratory, 2010. Technical Report NREL/TP-5000-48191.

[27] JM Jonkman, S Butterfield, W Musial, and G Scott. Definition of a 5-MW reference wind turbine for offshore system development, 2009. Technical Report NREL/TP-500-38060.

[28] A. M. Hansen and T. J. Larsen. Gear dynamics. Technical report, Risoe National Lab., 2009. Research in Aeroelasticity EFP-2007-II, Risoe-R-1698(EN):134-142.

[29] R Shirzadeh, C Devriendt, M Ahmadi Bidakhvidi, and P Guillaume. Experimental and computational damping estimation of an offshore wind turbine on a monopile foundation. Journal of Wind Engineering and Industrial Aerodynamics, 120:96-106, 2013.

[30] Christof Devriendt, Pieter Jan Jordaens, Gert De Sitter, and Patrick Guillaume. Damping estimation of an offshore wind turbine on a monopile foundation. IET Renewable Power Generation, 7(4):401-412, 2013. 